\title{
Treatment Outcomes in a Partial Hospital Program for Patients with Social Anxiety Disorder: The Effects of Comorbid Major Depression
}

\author{
Allison Graham, ${ }^{a,}$, Douglas R. Terrill, Simone I. Boyd ${ }^{h}$, Isabel Benjamin ${ }^{a}$, Madeline Ward ${ }^{h}$, \& Mark. Zimmerman ${ }^{a, c}$ \\ ${ }^{a}$ Rhode Island Hospital Department of Psychiatry, Providence, RI \\ ${ }^{b}$ Department of Cognitive, Linguistic, and Psychological Sciences, Brown University, Providence, RI \\ 'Department of Psychiatry and Human Behavior, Brown Alpert Medical School, Providence, RI \\ https:// doi.org/10.33697/ajur.2020.033 \\ Student:allison_graham@alumni.brown.edu* \\ Mentor:miimmerman@lifespan.org
}

\begin{abstract}
Although previous studies have examined the impact of comorbid major depressive disorder (MDD) on social anxiety disorder (SAD), the results have been somewhat mixed. Furthermore, most studies have been conducted in inpatient or outpatient settings. Given the large body of literature that suggests that this particular comorbidity can have negative effects on treatment efficacy and outcomes, it is important to continue to explore its impact. The present study aims to clarify contradictions in the literature and expands on previous studies by examining patients in a partial hospitalization setting. Patients at Rhode Island Hospital with a diagnosis of SAD were compared to those with comorbid SAD and MDD on pre-treatment and post-treatment measures of anxiety and depression. The results indicated that while the comorbid group showed significantly less improvement post-treatment on anxiety symptoms and constructs related to remission from depression, they did not show significantly less improvement on depression symptoms. The implications of these results for clinical practice are discussed.
\end{abstract}

\section{KEYWORDS}

Social Anxiety Disorder; Major Depressive Disorder; Comorbid Anxiety Disorders; Comorbid Mood Disorders; Treatment Outcomes; Partial Hospitalization Program

\section{INTRODUCTION}

Social anxiety disorder (SAD) is one of the most common mental disorders in the U.S., with a prevalence (12.1\%) behind only major depressive disorder (MDD; $16.6 \%$ ), alcohol use disorder (13.2\%), and specific phobias (12.5\%). ${ }^{1}$ According to the 2001 2003 National Comorbidity Survey, nearly one in 10 individuals in the U.S. suffer from SAD during their lifetime. ${ }^{2}$ Not only is SAD common, the disorder can have debilitating effects. Roughly one-third of people diagnosed with SAD in the U.S. report severe interpersonal, occupational, or psychosocial impairment. ${ }^{2}$ Over $90 \%$ of those diagnosed with SAD report some level of impairment in one or more of these domains. ${ }^{3}$ The pervasive, chronic, and disruptive nature of the disorder highlights a need for effective treatments.

A high rate of psychiatric comorbidity among those with SAD further increases the complexity and resulting impairment of this disorder. Prevalence estimates of comorbid disorders with SAD are as high as $90 \%$, with major depressive disorder (MDD) being the most common comorbid diagnosis, estimated in 30-75\% of individuals with SAD. ${ }^{4}$ A diagnosis of SAD has even been demonstrated to predict subsequent MDD, ${ }^{5}$ further highlighting the relationship between these disorders. This comorbidity results in much greater impairment across various domains than either disorder alone. Patients with comorbid SAD and MDD often experience more severe social anxiety symptoms, ${ }^{4}$ greater psychosocial impairment, longer depressive episodes, increased suicidal ideation, more time out of work, and greater likelihood of substance abuse. ${ }^{6}$ Greater impairment associated with this comorbidity may significantly affect the clinical course, underscoring a need for more research on treatment outcomes among this population.

Several treatments have been found to be efficacious in treating SAD or MDD. Cognitive Behavioral Therapy (CBT) is the most widely studied psychosocial approach to treating these disorders and produces consistently positive results in both group and individual settings. ${ }^{7-10}$ For patients with SAD, CBT shows nearly twice the response rate of placebo groups (50-56\% vs. 32\%) and remission rates between $8 \%$ and $36 \%$, indicating moderate short-term and long-term efficacy. ${ }^{10}$ Other psychosocial treatments that have demonstrated similar efficacy in treating these disorders include interpersonal therapy, psychodynamic therapy, and mindfulness-based treatments. ${ }^{10}$ Patients with either diagnosis have been shown to improve as a result of mindfulness-based stress 
reduction programs (MBSR), ${ }^{11-13}$ mindfulness-based cognitive therapy (MBCT), ${ }^{14-16}$ and Acceptance and Commitment Therapy (ACT). ${ }^{17-20}$ These results illustrate that a number of different therapies can successfully treat individuals with SAD or MDD.

While certain treatments are effective in treating SAD and MDD individually, there are mixed results on treatment outcomes among individuals with comorbid SAD and MDD. Some research has shown that the presence of this comorbidity does not produce different treatment outcomes than the presence of SAD or MDD alone. ${ }^{21}$ For instance, one study found that comorbid SAD did not interfere with CBT outcomes in depressed patients. ${ }^{22}$ Another preliminary study found that 16 sessions of ACT improved both symptoms and functioning in adults with SAD and MDD in an outpatient psychiatric setting. ${ }^{23}$ However, it is unclear whether these gains would be comparable to those obtained by patients with a single SAD diagnosis undergoing the same therapy. Conversely, some research has found poorer treatment outcomes for those with comorbid SAD and MDD. One study demonstrated that SAD patients with severe depressive symptoms, while not meeting clinical criteria for MDD, exhibited diminished response to treatment and increased treatment drop-out rates when compared to SAD patients with less severe depressive symptoms. ${ }^{24}$ Social anxiety symptoms in depressed patients treated with CBT and cognitive-behavioral group therapy (CBGT) have also been shown to increase during one-year follow-up periods. ${ }^{25}$ Other research on the efficacy of CBT has found smaller magnitudes of improvement in those with comorbid SAD and MDD than those with SAD alone. ${ }^{21}$ Given this conflicting evidence, further research into treatment outcomes for comorbid SAD and MDD is needed to clarify the response of these individuals to psychological treatment.

The majority of research evaluating treatment outcomes for patients with SAD or comorbid SAD and MDD has been conducted within inpatient and outpatient treatment settings ${ }^{26-27}$ Partial hospitalization programs fall between the differing intensities of these settings. While some research has shown CBT to be effective for either SAD or MDD in a partial hospital setting, there has been limited research conducted in this setting overall. ${ }^{28-29}$ The authors are not aware of any research evaluating differences in treatment outcomes in a partial hospital setting for individuals with SAD alone and individuals with comorbid SAD and MDD. The current study from the Rhode Island Hospital Methods to Improve Diagnostic and Assessment Services (MIDAS) Project aims to compare treatment outcomes between patients with a diagnosis of SAD in the absence of MDD and patients with comorbid SAD and MDD. While research on this topic is mixed, it was hypothesized that treatment outcomes would be poorer across a number of domains for those with comorbid SAD and MDD than those with SAD in the absence of MDD. Specifically, due to literature that has linked this comorbidity with poor treatment outcomes, ${ }^{21,24,25}$ it was expected that individuals with comorbid SAD and MDD would demonstrate less improvement during treatment, and upon discharge would exhibit more impairment in everyday life and greater severity of anxiety and depression when compared to individuals with SAD in the absence of MDD.

\section{METHODS AND PROCEDURES}

The Rhode Island Hospital institutional review committee approved the following research protocol, and all patients provided informed, written consent.

\section{Site}

Data was collected from individuals referred to the Rhode Island Hospital partial hospitalization program for first time treatment. The program treats patients five days per week, and patients attend four group therapies throughout the day, as well as daily individual meetings with a therapist and psychiatrist. Patients return home after each day in the program. Both group and individual therapies are largely based in Acceptance and Commitment Therapy. Patients undergo mindfulness training during treatment, and the program also offers a volunteer, therapist-led meditation group. Duration of treatment is most often based on treatment responses, thus, patients who improve more quickly discharge from the program after fewer days of treatment than those who do not. The average duration of treatment is just over eight days.

\section{Participants}

Between April 2014 and January 2020, 4,634 individuals presented for treatment to the Rhode Island Hospital Department of Psychiatry Partial Hospital Program. For patients who were treated in the program more than once, we included data only from their first admission. Patients were excluded if they had missing data on measures of depression or anxiety at baseline or discharge $(n=2,547)$. Patients were also excluded if they did not have a current diagnosis of social anxiety disorder $(n=1,617)$, leaving a sample of 470 patients. Diagnoses were made on patients' first day of attendance in the program, either by a trained clinical interviewer who administered the Structured Clinical Interview for ${\operatorname{DSM}-I^{30}}^{30}(n=292)$, or by a board-certified psychiatrist $(n=$ 178).

As the focus of this investigation concerned the impact of MDD on treatment outcomes in patients with a diagnosis of SAD, an MDD only comparison group was not included. Furthermore, our choice of a SAD comparison group was based on previous findings that a diagnosis of SAD precedes a diagnosis of MDD in $69 \%$ of comorbid cases, even predicting later development of 
MDD. ${ }^{5}$ Because patients with a SAD/MDD comorbidity are more likely to have been diagnosed with SAD first, comparing comorbid patients to those with SAD alone was most intuitive.

\section{Measures}

Clinically Useful Anxiety Outcome Scale: The Clinically Useful Anxiety Outcome Scale (CUXOS) ${ }^{31}$ is a 25 -item self-report measure of anxiety symptom severity. Patients are asked to rate the frequency of anxiety symptoms on an ordinal scale with the prompt "in the past week, including today..." (for the intake scale) and "during the past day..." (for the daily scale). Possible responses include (0) not at all, (1) a little bit, (2) a moderate amount, (3) quite a bit, and (4) almost always. The daily version of the CUXOS has demonstrated good internal consistency (Cronbach's alpha at intake $=0.93$; Cronbach's alpha at followup $=0.95)$, test-retest reliability ( $r=0.95$ and 0.96 , at intake and follow-up respectively), and was more correlated with measures of anxiety than with scales measuring other symptoms such as depression. ${ }^{32}$ First and last day scores were used from the CUXOS to represent pre- and post-treatment anxiety symptom severity.

Clinically Useful Depression Outcome Scale: The Clinically Useful Depression Outcome Scale (CUDOS) ${ }^{33}$ is an 18 -item selfreport measure of depression symptom severity. Patients are asked to rate the severity of depression symptoms "in the past week, including today..." (for the intake scale) and "during the past day..." (for the daily scale). Possible responses include (0) not at all, (1) a little bit, (2) a moderate amount, (3) quite a bit, and (4) almost always. The daily version of the CUDOS has been found to exhibit high internal consistency (Cronbach's alpha at intake $=0.82$, Cronbach's alpha at follow-up $=0.93)$, test-retest reliability, $(r$ $=0.91$ at intake, $r=0.98$ at follow-up) and high correlation with other measures of depressive symptoms. ${ }^{34}$ First and last day scores were used from the CUDOS to represent pre- and post-treatment depression levels in this study.

Remission from Depression Questionnaire: The Remission from Depression Questionnaire (RDQ) ${ }^{35}$ is a 60 -item measure designed to capture a broad array of domains considered by patients to be relevant to constructs of depression remission. These domains include symptoms (25 items), coping ability (five items), positive mental health (12 items), functioning (10 items), and wellbeing and life satisfaction (eight items). This scale has demonstrated good internal consistency (overall Cronbach's alpha $=$ 0.97, Cronbach's alpha for each subscale $>0.80)$, test-retest reliability $(r=0.85$ for overall scale, and $r>0.64$ for each subscale), and was significantly correlated with measures of depression, anxiety, psychosocial impairment, and quality of life..$^{35}$ As the CUDOS and CUXOS are more comprehensive measures of depression and anxiety symptom severity, the RDQ symptom subscale was not included in analysis.

\section{Analytic Strategy}

Patients included in analysis were divided into two groups: those with a current diagnosis of social anxiety disorder (SAD) in the absence of major depressive disorder (MDD) $(n=161)$, and those with comorbid SAD and MDD $(n=309)$. Demographic and clinical information were compared between groups, using Chi-square tests for categorical variables and t-tests for continuous variables. A series of one-way analysis of covariance models (ANCOVAs) were conducted to determine differences in measures of symptom and functioning change during treatment between groups, while controlling for score of pre-treatment measures. Levene's test and normality checks were carried out and the assumptions met.

\section{RESULTS}

Demographics

This sample was predominately white, identified as female, and had completed at least a high school education. Patients were between 18 and 80 years old $(M=33.3, S D=13.4)$, and patients in the comorbid SAD and MDD group were significantly older $(34.49 \pm 13.82$ vs. $31.03 \pm 12.26, p=.008)$. Full demographic results for each group can be found in Table 1.

\begin{tabular}{|l|c|c|c|c|c|c|}
\hline Demographic Characteristic & \multicolumn{2}{|c|}{ SAD (n = 161) } & \multicolumn{2}{c|}{ SAD/MDD (n = 309) } & \multicolumn{2}{c|}{ Group test } \\
\hline Gender & $\boldsymbol{N}$ & $\%$ & $\boldsymbol{N}$ & $\boldsymbol{x}$ & 1.84 & .40 \\
\hline Female & & & & & \\
\hline Male & 107 & 66.5 & 207 & 67.0 & \\
\hline Trans, Nonbinary & 44 & 27.3 & 91 & 28.5 & \\
\hline Race/Ethnicity & 10 & 6.2 & 11 & 3.6 & \\
\hline White & & & & & \\
\hline Black & 122 & 75.8 & 247 & 79.9 & & .42 \\
\hline
\end{tabular}




\begin{tabular}{|c|c|c|c|c|c|c|}
\hline Hispanic & 11 & 6.8 & 22 & 7.1 & & \\
\hline Asian & 8 & 5.0 & 7 & 2.3 & & \\
\hline Other & 7 & 4.3 & 10 & 3.2 & & \\
\hline Relationship Status & & & & & 8.19 & .15 \\
\hline Married & 24 & 14.9 & 66 & 21.4 & & \\
\hline Single & 98 & 60.9 & 152 & 49.2 & & \\
\hline Cohabiting & 15 & 9.3 & 36 & 11.7 & & \\
\hline Divorced/Separated & 17 & 10.6 & 38 & 12.3 & & \\
\hline Widowed & 1 & 0.6 & 8 & 2.6 & & \\
\hline Education & & & & & 3.53 & .90 \\
\hline High School Degree & 151 & 93.8 & 289 & 93.5 & & \\
\hline 4-Year College Degree & 49 & 30.4 & 82 & 26.5 & & \\
\hline \multirow[t]{2}{*}{ Graduate Degree } & 11 & 6.8 & 24 & 7.8 & & \\
\hline & $M$ & $S D$ & $M$ & $S D$ & $t$ & \\
\hline Age & 31.03 & 12.26 & 34.49 & 13.82 & -2.67 & .008 \\
\hline
\end{tabular}

Table 1. Demographic and Diagnostic Characteristics in Outpatient and Partial Hospital Samples

Clinical Differences

At baseline, patients in the comorbid group had significantly higher depression severity, fewer coping skills, lower functioning, poorer well-being, and less positive mental health than the SAD group. Conversely, there was no difference in baseline anxiety severity between the two groups. Full clinical differences between groups at baseline can be found in Table 2 .

\begin{tabular}{|c|c|c|c|c|c|c|c|}
\hline \multirow[t]{2}{*}{ Measure } & \multicolumn{3}{|c|}{ SAD } & \multicolumn{3}{|c|}{$\mathrm{SAD} / \mathrm{MDD}$} & \multirow[b]{2}{*}{$T$-Value } \\
\hline & $\mathbf{N}$ & $M$ & $S D$ & $\mathbf{N}$ & $M$ & $S D$ & \\
\hline CUDOS & 161 & 38.91 & 13.52 & 309 & 44.63 & 10.55 & $-5.05^{*}$ \\
\hline CUXOS & 161 & 40.43 & 16.84 & 309 & 41.60 & 16.32 & -0.72 \\
\hline Coping Skills & 141 & 3.24 & 2.19 & 277 & 2.39 & 2.04 & $3.93^{*}$ \\
\hline Functioning & 140 & 7.94 & 4.42 & 276 & 6.51 & 3.81 & $3.42 *$ \\
\hline Well-Being & 140 & 3.89 & 3.63 & 276 & 2.58 & 3.02 & $3.91 *$ \\
\hline Positive Mental Health & 140 & 7.31 & 5.40 & 277 & 4.29 & 4.08 & $6.39 *$ \\
\hline
\end{tabular}

Table 2. Baseline Characteristics Between SAD and SAD/MDD Groups. ${ }^{*} p<.05$

At discharge, however, the comorbid group had significantly higher anxiety severity than the SAD group. Once again, the comorbid group also had significantly higher depression severity, fewer coping skills, lower functioning, poorer well-being, and less positive mental health than the SAD group. Full clinical differences between groups at discharge can be found in Table 3 .

\begin{tabular}{|c|c|c|c|c|c|c|c|}
\hline \multirow[t]{2}{*}{ Measure } & \multicolumn{3}{|c|}{ SAD } & \multicolumn{3}{|c|}{ SAD/MDD } & \multirow[b]{2}{*}{$T$-Value } \\
\hline & $\boldsymbol{N}$ & $M$ & $S D$ & $\boldsymbol{N}$ & $M$ & $S D$ & \\
\hline CUDOS & 161 & 16.52 & 14.09 & 309 & 21.46 & 14.76 & $-3.50^{*}$ \\
\hline CUXOS & 161 & 21.01 & 17.40 & 309 & 25.15 & 18.19 & $-2.38^{*}$ \\
\hline Coping Skills & 102 & 6.13 & 2.59 & 209 & 4.59 & 2.48 & $5.05^{*}$ \\
\hline Functioning & 102 & 12.96 & 4.65 & 209 & 10.81 & 4.76 & $5.20 *$ \\
\hline Well-Being & 102 & 9.01 & 4.33 & 209 & 6.72 & 4.46 & $3.77 *$ \\
\hline $\begin{array}{l}\text { Positive Mental } \\
\text { Health }\end{array}$ & 102 & 14.44 & 5.53 & 209 & 10.81 & 5.88 & $4.29 *$ \\
\hline
\end{tabular}

Table 3. Discharge Clinical Characteristics Between SAD and SAD/MDD Groups. ${ }^{*} p<.05$ 


\section{Treatment Outcomes}

Number of days in treatment did not differ between groups $(7.78 \pm 5.05$ vs. $8.49 \pm 5.29, p=.16)$. Both the observed and adjusted means showed that the comorbid group was more impaired at discharge across all measures. At treatment termination, the comorbid group showed significantly less improvement in anxiety severity $(F(1,467)=5.52, p=.02)$, coping skills $(F(1,278)$ $=16.44, p<.01)$ functioning $(F(1,278)=4.43, p=.04)$, positive mental health $(F(1,278)=10.63$, $p=.001)$, and well-being and life satisfaction $(F(1,278)=6.54, p=.01)$ than the SAD group. The comorbid group did not significantly differ from the SAD group in terms of improvement in depressive symptom severity $(F(1,467)=3.62, p=.07)$. Although the comorbid group exhibited less improvement in anxiety symptoms and across RDQ domains relative to the SAD group, both groups improved greatly across all measures during treatment.

\section{DISCUSSION}

Consistent with literature that has found poorer treatment outcomes among those with comorbid SAD and MDD, our results suggest that comorbid patients exhibited less improvement in anxiety symptoms, coping skills, functioning, positive mental health, and well-being and life satisfaction than patients with SAD alone. On the other hand, our hypothesis that the comorbid group would show less improvement in depressive severity was not supported, with neither group showing significantly greater improvement in depressive symptoms.

These results are in line with literature that has found diminished treatment responses and smaller magnitudes of improvement among patients with comorbid SAD and MDD. ${ }^{21,24}$ The finding that anxiety symptoms improved comparatively less than depressive symptoms among comorbid patients is likewise supported by previous studies. In examining treatment outcomes among depressed patients with comorbid anxiety disorders, one study found that comorbid SAD uniquely produced greater reductions in depressive symptoms during treatment as well as elevated social anxiety symptoms during and after treatment. ${ }^{22}$ Another study also described increased social anxiety symptoms in patients with comorbid SAD and MDD following treatment. ${ }^{25}$ Insofar as our study supports these conclusions, our results indicate that anxiety symptoms should be a point of increased focus for clinicians when treating SAD patients with comorbid MDD.

It is also of note that while the amount of improvement in depressive symptom severity during treatment did not differ between the two groups, the comorbid group did show significantly less improvement in depression-adjacent constructs measured by the RDQ. Given that these constructs have been rated by patients as important in determining remission from depression, ${ }^{34}$ this indicates that those in the comorbid group derived less benefit from treatment than those in the SAD only group, even if the depressive symptoms themselves are not improving at significantly different rates.

Future research could expand upon our findings in a number of ways. Research building on differences found in this study between anxiety and depressive symptom improvement among those with comorbid SAD and MDD is warranted. Moscovich et al. reported that changes in social anxiety symptoms during CBGT account for $91 \%$ of changes in depressive symptoms, whereas changes in depression account for only $6 \%$ of changes in social anxiety symptoms. ${ }^{36}$ Thus, targeting social anxiety symptoms in patients with this comorbidity will likely simultaneously reduce depression. Other research enumerates which aspects of social anxiety may require more focus in therapy among patients with SAD/MDD. For instance, social avoidance has been shown to be augmented in comorbid patients both during and up to 32 months after treatment. ${ }^{22}$ Given Moscovitch et al.'s conclusions that changes in social anxiety mediate changes in depression among comorbid patients, ${ }^{36}$ future studies should examine symptom-level changes during treatment in order to assess which cognitive or behavioral areas exhibit differential improvement between these two disorders. Such research could reveal more precisely which specific social anxiety symptoms are associated with these changes in depression.

In addition, some research has suggested that gains made in therapy are typically not maintained over a one-year follow-up period in SAD patients with current MDD. ${ }^{25}$ Furthermore, current MDD predicted the post-treatment loss of gains better than the presence of other comorbid anxiety disorders or social anxiety severity at intake. ${ }^{25}$ Other researchers have found that depressive symptom severity, but not social anxiety symptom severity, at intake is related to poorer treatment response and higher drop-out rates. ${ }^{24}$ Due to these findings, future studies in partial hospital settings should examine whether improvements made during treatment are maintained after discharge.

Along similar lines, future research should explore treatment outcomes as a function of the duration of treatment. Research on the efficacy of acute partial hospital treatment is extremely limited and previous findings on the outcomes of shorter treatment durations are conflicted. Although most research has found acute treatment (5-8 days) in partial hospital settings to be comparable to inpatient treatment or longer partial hospital stays in treating psychiatric disorders, ${ }^{37,38,39}$ other research has suggested that shorter stays may have negative impacts on readmission rates ${ }^{40}$ and suicide risk ${ }^{41}$ among depressed patients. 
Data for the current study was collected in an in-person treatment format prior to the Covid-19 pandemic. It may be important to explore effects of the Covid-19 pandemic on psychiatric populations receiving care virtually in partial hospital programs. Patients with higher baseline symptom severity and greater numbers of psychiatric comorbidities, such as those typically treated in partial hospital programs, may experience more severe and persistent effects than others. However, a virtual treatment format requires less patient interaction, which may be beneficial to patients with a diagnosis of SAD. The impact of the pandemic on mental health may be long-lasting, and has magnified healthcare and economic disparities, disproportionately affecting ethnic and other minority populations. ${ }^{42}$ Exploration of treatment outcomes as a result of adaptations made to mental health care in the last year are warranted.

The current study has some important limitations. This study was conducted in a single clinical location in which the majority of patients were white, female, and had health insurance. Replication of this study in different treatment settings and a sample with different demographic characteristics is warranted. In addition, the CUXOS items are designed to assess general anxiety symptoms rather than specific social anxiety symptoms. Future extensions of this study may benefit from measures designed specifically to assess social anxiety. This study also chose to focus on differences in treatment outcomes exclusively among SAD patients with and without MDD. As SAD typically predates development of MDD in patients with both disorders, this study examined how comorbid MDD impacts treatment for patients with SAD, rather than the other way around. However, this line of research would benefit from the inclusion of an MDD group in the future, to determine if results would differ.

\section{CONCLUSIONS}

Despite these limitations, our research makes several contributions to existing literature. By exploring treatment outcomes in a partial hospital setting, this study expands upon and supports studies that have found poorer treatment outcomes among comorbid patients within inpatient and outpatient settings. In addition, this is the first study that we are aware of that examines these differences in a partial hospital setting. Furthermore, our results align with past findings regarding increased anxiety symptoms among comorbid patients, clarifying points for future research and indicating potential areas for clinician focus. Our research confirms that a comorbid diagnosis of SAD and MDD may present unique challenges that should be properly addressed in treatment and encourages further exploration of these challenges.

\section{REFERENCES}

1. Kessler, R. C., Berglund, P., Demler, O., Jin, R., Merikangas, K. R., and Walters, E. E. (2005) Lifetime prevalence and age-of-onset distributions of DSM-IV disorders in the national comorbidity survey replication, Arch Gen Psychiatry 63, 593602. https:/ / doi.org/10.1001/archpsyc.62.6.593

2. Stein, D.J., Lim, C.C.W., Roest, A.M., de Jonge, P., Aguilar-Gaxiola, S., Al-Hamzawi, A., Alonso, J., Benjet, C., Bromet, E. J., Bruffaerts, R., de Girolamo, G., Florescu, S., Gureje, O., Haro, J. M., Harris, M. G., He, Y., Hinkov, H., Horiguchi, I., Hu, C., Karam, A., Karam, E. G., Lee, S., Lepine, J.-P., Navarro-Mateu, F., Pennell, B.-E., Piazza, M., Posada-Villa, J., ten Have, M., Torres, Y., Viana, M. C., Wojtyniak, B., Xavier, M., Kessler, R.C., Scott, K. M., and WHO world Mental Health Survey Collaborators (2017) The cross-national epidemiology of social anxiety disorder: Data from the World Mental Health Survey Initiative, BMC Med 15, n.p. https:// doi.org/10.1186/s12916-017-0889-2

3. Ruscio, A.M., Brown, T.A., Chiu, W.T., Sareen, J., Stein, M.B., \& Kessler, R.C. (2008). Social fears and social phobia in the USA: Results from the National Comorbidity Survey Replication, Psychol Med 38, 15-28. https:// doi.org/10.1017/S0033291707001699

4. Koyuncu, A., Ince, E., Ertekin, E., and Tükel, R. (2019) Comorbidity in social anxiety disorder: Diagnostic and therapeutic challenges, Drugs Context 8, 1-13. https:// doi.org/10.7573/dic.212573

5. Bittner, A., Goodwin, R. D., Wittchen, H.-U., Beesdo, K., Höfler, M., \& Lieb, R. (2004) What characteristics of primary anxiety disorders predict subsequent major depressive disorder?, J Clin Psychiatry 65, 618-626. https://doi.org/10.4088/jcp.v65n0505

6. Dalrymple, K. L. and Zimmerman, M. (2007) Does comorbid social anxiety disorder impact the clinical presentation of principal major depressive disorder? J Affect Disord 100, 241-247. https:// doi.org/10.1016/j.jad.2006.10.014

7. Heimberg, R. G. (2002) Cognitive-behavioral therapy for social anxiety disorder: Current status and future directions, Biol Psychiatry 51, 101-108. https:/ / doi.org/10.1016/s0006-3223(01)01183-0

8. Hofmann, S. G., Asnaani, A., Vonk, I. J. J., Sawyer, A. T., and Fang, A. (2012) The efficacy of cognitive behavioral therapy: A review of meta-analyses, Cognit Ther Res 36, 427-440. https:/ / doi.org/10.1007/s10608-012-9476-1

9. Scaini, S., Belotti, R., Ogliari, A. and Battaglia, M. (2016) A comprehensive meta-analysis of cognitive behavioral interventions for social anxiety disorder in children and adolescents, J Anxiety Disord 42, 105-112.

https:// doi.org/10.1016/j.janxdis.2016.05.008.

10. Leichsenring, F. and Leweke, F. (2017) Social anxiety disorder, N Engl J Med 376, 2255-2264. https://doi.org/10.1056/NEJMcp1614701

11. Goldin, P. R. and Gross, J. J. (2010) Effects of mindfulness-based stress reduction (MBSR) on emotion regulation in social anxiety disorder, Emotion 10, 83-91. https://doi.org/10.1037/a0018441 
12. Hjeltnes, A., Molde, H., Schanche, E., Vøllestad, J., Lillebostad Svendsen, J., Moltu, C., and Binder, P.-E. (2017) An open trial of mindfulness-based stress reduction for young adults with social anxiety disorder, Scand J Psychol 58, 80-90. https://doi.org/10.1111/sjop.12342

13. Chi, X., Bo, A., Liu, T., Zhang, P., and Chi, I. (2018) Effects of mindfulness-based stress reduction on depression in adolescents and young adults: A systematic review and meta-analysis, Front Psychol 9, n.p. bttps: / / doi.org/10.3389/fpsyg.2018.01034

14. Strege, M. V., Swain, D., Bochicchio, L., Valdespino, A., and Richey, J. A. (2018) A pilot study of the effects of mindfulnessbased cognitive therapy on positive affect and social anxiety symptoms, Front Psychol 9, n.p. https:// doi.org/10.3389/fpsyg.2018.00866

15. Piet, J., Hougaard, E., Hecksher, M. S., and Rosenberg, N. K. (2010) A randomized pilot study of mindfulness-based cognitive therapy and group cognitive-behavioral therapy for young adults with social phobia, Scand J Psychol 51, 403-410. bttps://doi.org/10.1111/j.1467-9450.2009.00801.x

16. Goldberg, S. B., Tucker, R. P., Greene, P. A., Davidson, R. J., Kearney, D. J., and Simpson, T. L. (2019) Mindfulness-based cognitive therapy for the treatment of current depressive symptoms: A meta-analysis, Cogn Behav Ther 48, 445-462. bttps:/ / doi.org/ 10.1080/16506073.2018.1556330

17. Roohi, R., Soltani, A. A., Meimand, Z. Z., and Nematollahi, V. R. (2019) The effect of acceptance and commitment therapy (ACT) on increasing the self-compassion, distress tolerance, and emotion regulation in students with social anxiety disorder, Journal Child Ment Health 6, 173-187. https:/ / doi.org/10.29252/jcmh.6.3.16

18. Dalrymple, K. L. and Herbert, J. D. (2007) Acceptance and commitment therapy for generalized social anxiety disorder, Behav Modif 31, 543-568. https:// doi.org/10.1177/0145445507302037

19. Ivanova, E., Lindner, P., Ly, K. H., Dahlin, M., Vernmark, K., Andersson, G., and Carlbring, P. (2016) Guided and unguided acceptance and commitment therapy for social anxiety disorder and/or panic disorder provided via the Internet and a smartphone application: A randomized controlled trial, J Anxiety Disord 44, 27-35. bttps://doi.org/10.1016/j.janxdis.2016.09.012

20. Bai, Z., Luo, S., Zhang, L., Wu, S., and Chi, I. (2020) Acceptance and commitment therapy (ACT) to reduce depression: A systematic review and meta-analysis, J Affect Disord 260, 728-737. https:// doi.org/10.1016/j.jad.2019.09.040

21. Bauer, I., Wilansky-Traynor, P., and Rector, N. A. (2012) Cognitive-behavioral therapy for anxiety disorders with comorbid depression: A review, Intl J Cog Ther 5, 118-156. https://doi.org/10.1521/ijct.2012.5.2.118

22. Vittengl, J. R., Clark, L. A., Smits, J. A. J., Thase, M. E., and Jarrett, R. B. (2019) Do comorbid social and other anxiety disorders predict outcomes during and after cognitive therapy for depression?, J Affect Disord 242, 150-158. bttps:/ / doi.org/10.1016/j.jad.2018.08.053

23. Dalrymple, K. L., Morgan, T. A., Lipschitz, J. M., Martinez, J. H., Tepe, E., and Zimmerman, M. (2014) An integrated, acceptance-based behavioral approach for depression with social anxiety: Preliminary results, Behav Modif 38, 516-548. bttps://doi.org/10.1177/0145445513518422

24. Ledley, D.R., Huppert, J.D., Foa, E.B., Davidson, J.R., Keefe, F.J., and Potts, N.L (2005) Impact of depressive symptoms on the treatment of generalized social anxiety disorder, Depress Anxiety 22, 161-167. https://doi.org/10.1002/ da.20121

25. Marom, S., Gilboa-Schechtman, E., Aderka, I.M., Weizman, A., and Hermesh, H. (2009) Impact of depression on treatment effectiveness and gains maintenance in social phobia: a naturalistic study of cognitive behavior group therapy, Depress Anxiety 26, 289-300. https:/ / doi.org/10.1002/da.20390

26. McLean, P. D. and Hakstian, A. R. (1979) Clinical depression: Comparative efficacy of outpatient treatments, J Consult Clin Psychol 47, 818-836. https:// doi.org/10.1037/0022-006X.47.5.818

27. Cuijpers, P., Clignet, F., van Meijel, B., van Straten, A., Li, J., and Andersson, G. (2011) Psychological treatment of depression in inpatients: A systematic review and meta-analysis, Clin Psychol Rev 31, 353-360. https:// doi.org/10.1016/j.cpr.2011.01.002

28. Beard, C., Stein, A. T., Hearon, B. A., Lee, J., Hsu, K. J., and Björgvinsson, T. (2016) Predictors of depression treatment response in an intensive CBT partial hospital, J Clin Psychol 72, 297-310. https:/ / doi.org/10.1002/jclp.22269

29. Peckham, A. D., Forgeard, M., Hsu, K. J., Beard, C., and Björgvinsson, T. (2019) Turning the UPPS down: Urgency predicts treatment outcome in a partial hospitalization program, Compr Psychiatry 88, 70-76. https:/ / doi.org/10.1016/j.comppsych.2018.11.005

30. First M.B. (1997) A guide for the Structured Clinical Interview for DSM-IV axis 1 disorders SCID-1: Clinician version, American Psychiatric Press, Washington D.C.

31. Zimmerman, M., Chelminski, I., Young, D., and Dalrymple, K. (2010) A clinically useful anxiety outcome scale, J Clin Psychiatry 71, 534-542. http:// doi.org/10.4088/JCP.09m05264blu

32. Zimmerman, M., Kiefer, R., Kerr, S., and Balling, C. (2019) Reliability and validity of a self-report scale for daily assessments of the severity of anxiety symptoms, Compr Psychiatry 90, 37-42. https:/ / doi.org/10.1016/j.comppsych.2018.12.014

33. Zimmerman, M., Chelminski, I., McGlinchey, J. B., and Posternak, M. A. (2008) A clinically useful depression outcome scale, Compr Psychiatry 49, 131-140. https:// doi.org/ 10.1016/j.comppsych.2007.10.006

34. Zimmerman, M., Harris, L., Martin, J., and McGonigal, P. (2018) Reliability and validity of a self-report scale for daily assessments of the severity of depressive symptoms, Psychiatry Res 270, 581-586. https:// doi.org/ 10.1016/j.psychres.2018.10.007 
35. Zimmerman, M., Martinez, J. H., Attiullah, N., Friedman, M., Toba, C., Boerescu, D. A., and Ragheb, M. (2013) A new type of scale for determining remission from depression: The Remission from Depression Questionnaire, J Psychiatr Res 47, 78-82. https://doi.org/10.1016/j.jpsychires.2012.09.006

36. Moscovitch, D. A., Hofmann, S. G., Suvak, M. K., and In-Albon, T. (2005) Mediation of changes in anxiety and depression during treatment of social phobia, J Consult Clin Psychol 73, 945-952. https:/ / doi.org/ 10.1037/0022-006X.73.5.945

37. Morgan, T. A., Dalrymple, K., D’Avanzato, C., Zimage, S., Balling, C., Ward, M., \& Zimmerman, M. (2020) Conducting outcomes research in a clinical practice setting: The effectiveness and acceptability of acceptance and commitment therapy (ACT) in a partial hospital program, Behav Ther. https:// doi.org/10.1016/j.beth.2020.08.004

38. Lieberman, P. B., Villalba, R., \& Farris, S. G. (2017) Outcomes of acute partial hospital treatment. J Psychiatr Pract 23, $401-408$. bttps:// doi.org/ 10.1097/pra.0000000000000271

39. Larivière, N., Desrosiers, J., Tousignant, M., \& Boyer, R. (2011) Multifaceted impact evaluation of a day hospital compared to hospitalization on symptoms, social participation, service satisfaction and costs associated to service use, Int J Psychiatry Clin Pract 15, 228-240. https: / / doi.org/10.3109/13651501.2011.572170

40. Figueroa, R., Harman, J., \& Engberg, J. (2004) Use of claims data to examine the impact of length of inpatient psychiatric stay on readmission rate, Psychiatr Serv 55, 560-565. https:// doi.org/10.1176/appi.ps.55.5.560

41. Desai, R. A., Dausey, D. J., \& Rosenheck, R. A. (2005) Mental health service delivery and suicide risk: The role of individual patient and facility factors, Am J Psychiatry 162, 311-318. https:// doi.org/10.1176/appi.ajp.162.2.311

42. Moreno, C., Wykes, T., Galderisi, S., Nordentoft, M., Crossley, N., Jones, N., . . Arango, C. (2020) How mental health care should change as a consequence of the COVID-19 pandemic, Lancet Psychiatry 7, 813-824. https://doi.org/10.1016/s2215-

0366(20)30307-2

\section{ABOUT STUDENT AUTHOR}

Allison Graham graduated magna cum laude from Brown University in 2020. She plans on pursuing a PhD in clinical psychology and a career as a licensed psychologist.

\section{PRESS SUMMARY}

Although previous studies have examined the impact of comorbid major depressive disorder (MDD) on social anxiety disorder (SAD), the results have been somewhat mixed. Most studies have found that comorbid MDD negatively impacts patients with SAD both in terms of everyday impairment and in terms of treatment efficacy and outcomes. This study confirms these findings, while examining this comorbidity in an under-studied setting (partial hospitalization program). Patients at Rhode Island Hospital with a diagnosis of SAD were compared to those with comorbid SAD and MDD on pre-treatment and post-treatment measures of anxiety and depression. As predicted, the comorbid groups showed significantly less improvement on anxiety symptoms and several constructs related to remission from depression. However, there were no significant differences on improvement in depressive symptoms. These findings corroborate previous studies which have suggested that anxiety symptoms may need to be a point of focus for patients with this comorbidity. 